\title{
A SIMPLE PROOF OF A THEOREM OF ISBELL
}

A. KRUSE

John Isbell [3, pp. 301-302] proved the following theorem. ${ }^{1,2}$

THEOREM. Every complete metric space is homeomorphic with a closed subset of a countable product of finite-dimensional uniform complexes. ${ }^{3}$

We shall give a simple proof of this theorem utilizing embeddings in Hilbert space.

Consider any real Hilbert space $H$. Let $E$ be an orthonormal basis of $H$; let $\theta$ be the origin of $H$. Let $E^{\prime}=E \cup\{\theta\}$. Let $|K|$ be the algebraic convex hull of $E^{\prime}$, whence $|K| \subset H$. We consider $|K|$ to be the underlying space of an obvious geometric simplicial complex $K$ whose set of vertices is $E^{\prime}$. Let $K^{(n)}$ be the $n$-skeleton of $K$; it is easily verified that $K^{(n)}$ as a uniform complex and $\left|K^{(n)}\right|$ as a topological subspace of $H$ are topologically the same. Let $Z$ be the closure of $|K|$ in $H$, whence $Z$ is the set of all $v \in H$ such that $v=\sum_{e \in E} t_{e} e$ and $\sum_{e \in E} t_{e} \leqq 1$ for some nonnegative scalars $t_{e}$.

Lemma 1. $Z$ is homeomorphic to a closed subset of $\prod_{n=0}^{\infty}\left|K^{(n)}\right|$.

Proof. Define $f_{n}: Z \rightarrow\left|K^{(n)}\right|$ as follows. If $v=\sum_{e \in E} t_{e} e$ as above, then, where $s_{e}$ is the smaller of $t_{e}$ and $(n+1)^{-1}$, let $f_{n}(v)=\sum_{e \in E}\left(t_{e}-s_{e}\right) e$. Define $f: Z \rightarrow \prod_{n=0}^{\infty}\left|K^{(n)}\right|$ so that $f_{n}$ is the $n$-coordinate function of $f$ for each $n$. It is easily shown that $f$ is a homeomorphism from $Z$ onto a closed subset of $\prod_{n=0}^{\infty}\left|K^{(n)}\right|$.

Lemma 2. Suppose that $(X, d)$ is a metric space and the orthonormal dimension of $H$ is as large as the cardinal of some base of $X$. Then there is a homeomorphism $g$ from $X$ into $Z$ (defined as above) such that $g^{-1}: g[X] \rightarrow X$ is a uniformly continuous. Hence, if $(X, d)$ is complete, then $X$ is homeomorphic to a closed subset of $Z$ (and thus to a closed subset of $H$ ).

Proof. After changing several obvious coefficients $n^{-1}$ to (for

Received by the editors May 30, 1966.

1 The question raised by the writer in his Zentralblatt (Vol. 119, pp. 181-182) review of [3] concerning the proof of this theorem arose from a notational misinterpretation; the proof in [3] is complete as it stands. The dimension $\Delta d$ studied in [3] should have been attributed to [2] in the same review. The writer regrets these inaccuracies.

${ }^{2}$ Isbell [3] uses this theorem to obtain a much sharper result.

${ }^{3}$ A uniform complex is metrized by the maximum difference of barycentric coordinates. 
example) $2^{-n}$ in $[1$, pp. 194-196], apply $[1,9.4$, p. 196] and its proof. Thus Lemma 2 comes from scrutinizing a standard proof of the Nagata-Smirnov metrization theorem.

Finally, the theorem follows immediately from Lemmas 1 and 2 .

\section{REFERENCES}

1. J. Dugundji, Topology, Allyn and Bacon, Boston, Mass., 1966.

2. J. R. Isbell, Zero-dimensional spaces, Tôhoku Math. J. (2) 7 (1955), 1-8.

3. - On finite-dimensional uniform spaces. II, Pacific J. Math. 12 (1962), 291-302.

New Mexico State University 